\title{
Depletion influences restraint, but does it influence conflict identification? Expanding on Osgood and Muraven (2015)
}

\author{
Kristian Ove R. Myrseth, University of St Andrews ${ }^{1}$ \\ Conny Wollbrant, University of Gothenburg ${ }^{2}$
}

\begin{abstract}
Osgood and Muraven (2015) show that cognitive depletion reduces pro-social behaviors, but not pro-social attitudes. We expand on the authors' interpretation by relating their results to recent theorizing on the relationship between pro-social behavior and self-control. This framework distinguishes between the proclivity to identify self-control conflict and the capacity to exercise restraint. Osgood and Muraven's (2015) findings can be interpreted as evidence that cognitive depletion in social contexts fails to influence a necessary condition for identifying self-control conflict. However, the results do not yet allow us to conclude that depletion influences capacity to exercise restraint. Further work is needed to understand the mechanisms by which cognitive depletion influences pro-social behavior.
\end{abstract}

Keywords: Pro-social behavior, Self-control, Depletion

\footnotetext{
${ }^{1}$ School of Management, University of St Andrews, The Gateway, North Haugh, St Andrews, Fife, KY16 9RJ. Scotland; e-mail: kom@st-andrews.ac.uk; phone: +44 1334 461972; e-mail: kom@standrews.ac.uk.

${ }^{2}$ Department of Economics, School of Business, Economics and Law, University of Gothenburg, Box 640, 40530 Gothenburg, Sweden; phone +46 3178626 15; e-mail: conny.wollbrant@economics.gu.se.
} 
Osgood and Muraven (2015) report that ego depletion reduces pro-social behavior in two economic games - a centipede game and a repeated game of extraction. However, they find no effect of depletion on players' pro-social attitudes. It is this joint resultego depletion affects pro-social behavior without affecting pro-social concerns - that represents the key contribution of their paper. We expand on the significance of their results by relating the pattern to recent theorizing about pro-social behavior and the two-stage model of self-control, which distinguishes between identifying self-control conflict and exercising restraint (Martinsson, Myrseth, \& Wollbrant, 2012; Kocher, Martinsson, Myrseth, \& Wollbrant, 2013; Martinsson, Myrseth, \& Wollbrant, 2014).

The two-stage model of self-control posits that an individual in the face of a tempting course of action, before drawing on mental tactics to exercise self-control, must identify whether a self-control problem has presented itself (Myrseth \& Fishbach, 2009). This postulate bears two important implications: (1) any act of selfrestraint implies that the individual at some level has recognized the self-control problem, and (2) the act of yielding to temptation could result either from failed resistance or from the failure to see the self-control problem in the first place. Thus, to understand when and why individuals engage in self-control, we must understand not only how they exercise restraint, but also when they see the self-control problem. It is on the basis of this framework that we evaluate how Osgood and Muraven (2015) speak to the role of ego depletion in pro-social behavior.

Ego depletion is known to reduce acts of self-control, but does it operate by weakening the capacity to exercise restraint, or by impairing the proclivity to identify the self-control problem? Recent work by Hedgcock, Vohs, and Rao (2012) points to the former mechanism over the latter, but this was in a different domainhypothetical choice of consumer goods. Osgood and Muraven (2015) present consistent results in the context of economic games: ego depletion reduced pro-social behavior, but not pro-social attitudes. It is worth pausing at this observation. Can we from the perspective of the two-stage model infer that ego-depletion in their economic games impaired the capacity to exercise restraint, but not the proclivity to identify self-control conflict? We cannot, although their results do shed light on the issue.

Osgood and Muraven (2015) interpret their results to mean that ego depletion has not altered pro-social concerns. That case is firm, and pro-social concerns - in this 
context-represent a necessary condition for identifying self-control conflict. However, such attitudes are not sufficient. It is possible that depleted individuals, although sharing pro-social attitudes with their non-depleted peers, nevertheless failed to see a connection between their current behavior and their pro-social concerns. To use the phrasing of Messick (1999), perhaps ego-depletion reduced the tendency for individuals to ask themselves the question, "what does a person like me do in a situation like this?" Perhaps ego depletion caused individuals to focus more on the unique situation at hand, rather than relating the situation to the more abstract class of past and future behavior that defines "what a person a like me" "is'? Martinsson et al. $(2012$; 2014) argue that the former perceptual frame is less likely than the latter to prompt identification of self-control conflict in the dictator and public good games. We raise these possibilities not because we believe they are more plausible than alternative accounts, but because we fail to see why they are substantially less plausible.

It would be premature to conclude that ego-depletion in Osgood and Muraven (2015) has influenced capacity to exercise restraint, as opposed to identification of self-control conflict. The possibility of the latter mechanism remains viable. However, the authors have demonstrated that a necessary condition for identifying conflictpro-social attitudes-remained intact in individuals subject to ego depletion. This finding is notable in light of Hedgcock et al. (2012), who show that depletion reduces activation in the dorsolateral prefrontal cortex-the region associated with implementation of executive control (MacDonald, Cohen, Stenger, \& Carter, 2000) but not in the anterior cingulate cortex-which is associated with conflict identification (MacDonald et al., 2000). The balance of evidence, then, suggests that ego depletion in general influences the capacity for restraint, not the proclivity for conflict identification - though this has yet to be demonstrated conclusively in the context of pro-social behavior. 
Depletion influences restraint, but does it influence conflict identification?

\section{References}

Hedgcock, W. M., Vohs, K. D., \& Rao, A. R. (2012). Reducing self-control depletion effects through enhances sensitivity to implementation: Evidence from fMRI and behavioral studies. Journal of Consumer Psychology, 22, 486-495.

Kocher, M. G., Martinsson, P., Myrseth, K. O. R., Wollbrant, C. E. (2013). Strong, bold and kind: Self-control and cooperation in social dilemmas. CESifo Working Paper No 4200, University of Munich.

MacDonald, A. W., III, Cohen, J. D., Stenger, V. A., \& Carter, C. S. (2000). Dissociating the role of the dorsolateral prefrontal and anterior cingulate cortex in cognitive control. Science, 288, 1835-1838.

Martinsson, P, Myrseth, K. O. R., \& Wollbrant, C. E. (2014). Social dilemmas: When self-control benefits cooperation. Journal of Economic Psychology, 45, 213236.

Martinsson, P., Myrseth, K. O. R., \& Wollbrant, C. E. (2012). Reconciling pro-social vs. selfish behavior: On the role of self-control. Judgment and Decision Making, 7: 304-315.

Messick, D. (1999). Alternative logics for decision making in social settings. Journal of Economic Behavior and Organization, 39: 11-28.

Myrseth, K. O. R., \& Fishbach, A. (2009). Self-control: A function of knowing when and how to exercise restraint. Current Directions in Psychological Science, 18: $247-252$.

Osgood, J. M., \& Muraven, M. (2015). Self-Control depletion does not diminish attitudes about being prosocial but does diminish prosocial behaviors. Basic and Applied Social Psychology, 37: 68-80. 